\title{
PHASE CORRECTION IN HOLOGRAPHICALLY PRODUCED GRATINGS
}

\author{
R. ALFERNESS \\ The University of Michigan, Ann Arbor, Michigan 48107, USA \\ Received 16 May 1974 \\ Revised manuscript received 6 August 1974
}

\begin{abstract}
Several techniques for producing holographic optical elements that are free from the inherent phase errors of their substrate irregularities are given.
\end{abstract}

Recently a method was described for producing holographic optical elements, such as gratings or zone plates, which are free of the inherent phase errors caused by substrate irregularities [1]. The procedure is to incorporate the appropriate correction factor into the recorded fringe structure during the interferometric construction of the grating. The method is useful for producing high quality holographic optics on inexpensive substrates. Here we extend the method to include compensation for arbitrarily large phase errors, give an alternate two-step holographic method and describe a technique which may be used in a system of successive gratings to compensate for the phase errors in more than a single order. We also describe how the latter technique may be used to make a well-corrected, yet inexpensive, grating interferometer. The mathematical analysis of phase correction, previously described, will not be repeated here [1].

The previous method requires that the phase error be sufficiently small so that in propagating from the substrate to a mirror and back, the phase error is unchanged. In practice this condition is at best only approximated, with the result that the recorded phase error is slightly different from that produced by the substrate, and the compensation is imperfect. This slight residual error in the compensated wavefront produces, in a shearing interferometer, fringes that are straight but slightly tilted with respect to the vertical direction [1]. (The shearing interferometer used was oriented so that straight, vertical fringes indicate a perfectly collimated beam.)

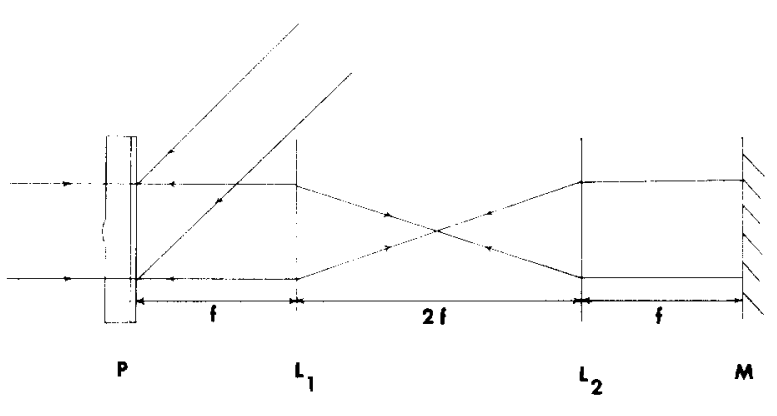

Fig. 1. Method for making self-corrected gratings. P, photographic plate with irregular substrate; $L_{1}$ and $L_{2}$, lenses of focal length $f$; and $\mathrm{M}$, mirror.

To produce complete compensation, regardless of the magnitude of the phase error, we have added a twolens imaging system to our phase correction arrangement (fig. 1). The aberrated wave leaving the emulsion is faithfully imaged back onto the emulsion provided well-corrected lenses are used. Here the wave interferes with a collimated reference wave to form a holographic grating. Illuminating the developed plate with a collimated plane wave I (fig. 2), produces a zero-order wave $D_{0}$ which acquires the aberration of the substrate and one diffracted first order wave $D_{-1}$ which has twice the phase error of the substrate. However, the conjugate wave $D_{+1}$ is free from the phase aberration [1]. The improvement of the above system in phase correction is shown in the experimental results of fig. 3 . The incident (fig. 3a), zero order (fig. 3c) and corrected first order (fig. $3 \mathrm{~b}$ ) are shown as viewed by a shearing inter- 


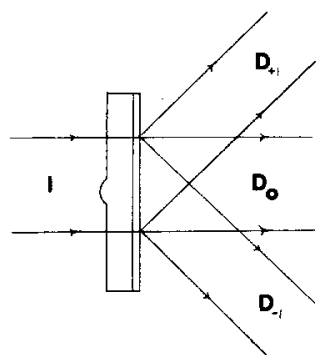

Fig. 2. Reconstruction using the self-corrected grating. I, incident collimated beam; $D_{0}$, zero-order beam aberrated by the substrate irregularity; $D_{+1}$ and $D_{-1}$, the first order diffracted beams, one of which is free from aberration.

ferometer. The incident beam is a collimated wave producing straight, vertical fringes. The zero order shows the aberration of the substrate. Correction in the first order is complete, as indicated by the straight and perfectly vertical fringes.

The technique described above requires well-corrected lenses and a high quality mirror. An alternate twostep holographic method which provides for exact phase correction without the need of lenses or a mirror is shown in fig. 4. In the first step a hologram of the phase error of plate $P_{1}$ is made on plate $P_{2}$ using collimated beams $A$ and $B$. Plate $P_{2}$ is developed, returned and read out with collimated beam $C$ which propagates in the direction opposite from $\mathrm{B}$. The exact conjugate phase error of $P_{1}$ is imaged onto itself and interferes with the collimated beam $\mathrm{D}$, forming a self-corrected grating. Again the grating is read out as shown in fig. 2 . That the conjugate rather than the actual phase error is imaged onto the emulsion during the construction process is unimportant for thin emulsion plates and merely results in the opposite order being the compensated one. All experimental results given here are for thin emulsions.

For thick emulsion gratings, however, the use of the conjugate phase in the method described above is very desirable and theoretically results in more complete phase correction than the use of the actual phase error. Thick emulsion gratings have only a single intense first order and we want that order to be the corrected one. When the actual phase error serves as the signal beam, the conjugate order is the corrected one [1]. However, the conjugate wave must be read out from
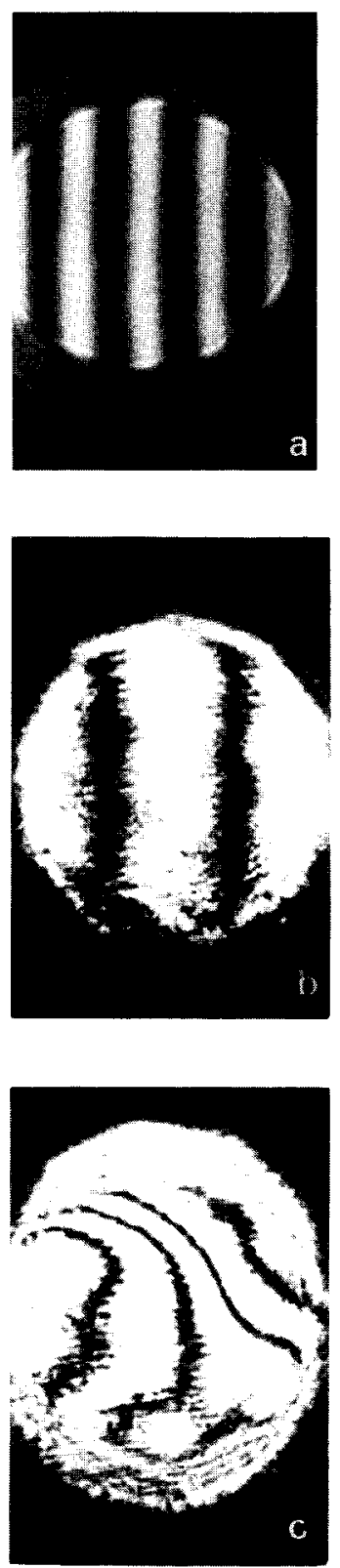

Fig. 3. Experimental results using the self-correction system of fig. 1. (a) incident beam; (b) corrected first order; (c) aberrated zero order as viewed by a shearing interferometer.

a thick grating by a beam that duplicates the construction signal beam (minus the phase error) which impinge at normal incidence on the emulsion side of the plate (beam $\mathrm{D}_{0}$ of fig. 2 , except traveling in the opposite direction). As a result the diffracted rather than the inci- 


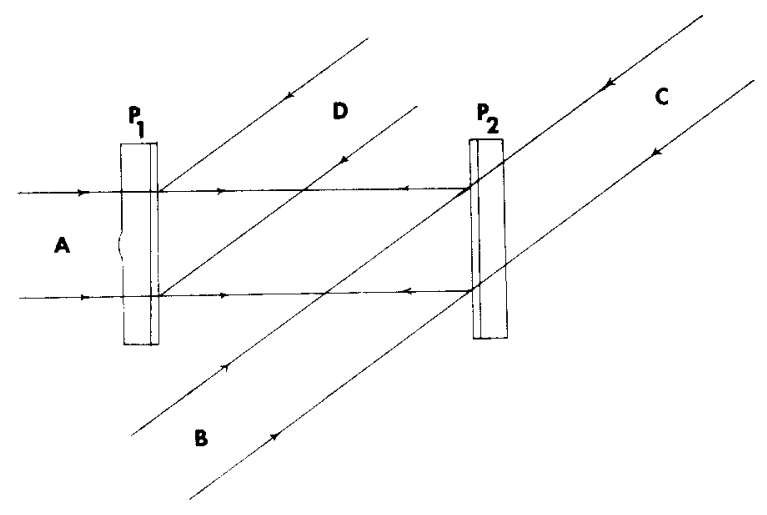

Fig. 4. Two-step holographic method for making self-corrected gratings. $P_{1}$ is plate on which grating is made. $P_{2}$ is used to make hologram of $P_{1}$. All beams are collimated.

dent wave passes through the substrate irregularity. Since the spatial frequency of the diffracted wave is different from that of the wave that encountered the irregularity during construction, then the diffracted beam will have a slightly different phase error than that corrected for in the recorded fringe structure and compenstation will be incomplete. When the signal beam contains the conjugate of the substrate phase error, however, the virtual order is the corrected one. For thick holograms the virtual order is read out by a beam duplicating the signal beam but of opposite direction (beam I of fig. 2) [2]. In this case the beam that passes through the substrate irregularity during reconstruction is identical to that used during construction. Therefore phase compensation will be complete in the strong order. This effect will generally be small except for high frequency gratings with rapidly varying substrate irregularities, in which case the method of fig. 4 offers definite advantage.

To achieve good correction, plate $\mathrm{P}_{2}$ (fig. 4) must be placed in a liquid gate during the construction process. The speed of the photosensitive plate $\mathrm{P}_{2}$ should be sufficiently greater than that of $P_{1}$ so that it is not overexposed while making the first hologram. Preliminary experimental results verify the soundness of the approach. Unfort unately, when using photographic film (Kodak 649F) noise buildup during the two steps is severe and limits the usefulness of the method. However, with less noisy photosensitive materials like dichromated gelatin such problems could probably be eliminated.

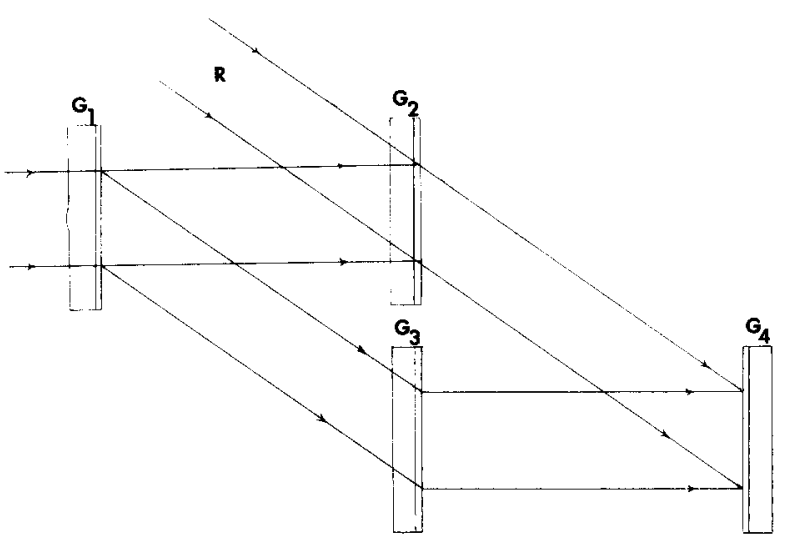

Fig. 5. Grating interferometer with phase correction. All gratings are of the same spatial frequency.

It is sometimes necessary to use more than a single order from a diffraction grating as, for example, when it is used as the beam splitter of an interferometer [3] . Unfortunately, all self-correcting techniques are limited to a single non-zero order. However, in the case of a grating interferometer where the elements are fixed in position, the phase error in the uncorrected order of the beam splitter can be corrected by a subsequent, compensated grating. Fig. 5 shows a typical configuration of the grating interferometer. The beam splitter $G_{1}$ is self-corrected for the minus first order. The phase error of the zero order of $G_{1}$ can be removed by a properly constructed grating $G_{2} . G_{2}$ is produced by interfering the aberrated zero order wave from $G_{1}$ with a collimated reference $R$. The plate is developed and returned and beam $\mathrm{R}$ is removed. The desired first order from $G_{2}$ (fig. 5 ) is then free from the aberration of the substrate of $G_{1}$. Obviously the self-correction technique can be included to compensate at the same time for the irregularities of $G_{2}$. Grating $G_{3}$ of the interferometer in fig. 5 can also be made self-correcting so that the interference fringes formed at the plane of $G_{4}$ in the absence of any object are perfectly straight and parallel. Placing the grating $\mathrm{G}_{4}$ (of same spatial frequenc as the other gratings) in the plane of these fringes produces Moiré fringes (of sufficiently low frequency to be easily visible) which are not sensitive to the possible substrate irregularities of $\mathrm{G}_{4}$. The grating interferometer can therefore be made with inexpensive elements and yet be fully corrected. Of course the correction is perfect only for the construction wavelength and incident 

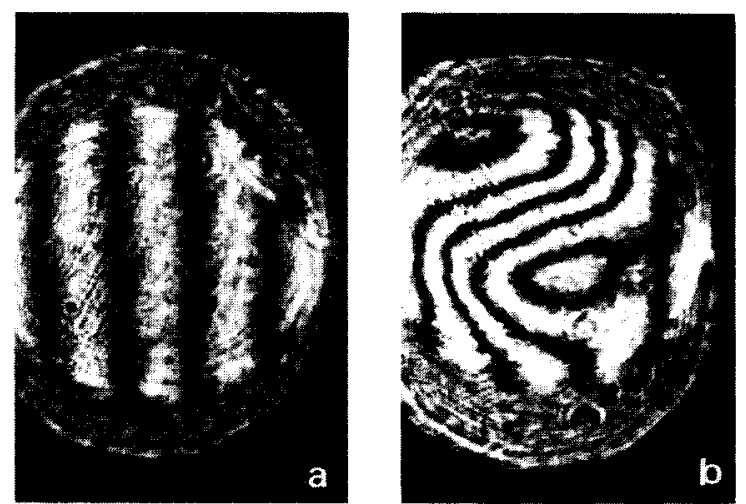

Fig. 6. Experimental results using system of fig. 5. (a) corrected first order from $\mathrm{G}_{2}$; (b) aberrated zero order from $\mathrm{G}_{2}$ as viewed by a shearing interferometer.

angle at $\mathrm{G}_{1}$, but reasonably good correction can be expected for narrow spectral and spatial bandwidths about these values. The accuracy with which $\mathrm{G}_{2}$ must be returned is quite critical in the lateral direction (perpen- dicular to the optical axis) depending on the frequency of the irregularity of $G_{1}$. It is much less critical in the longitudinal direction unless the grating frequency is very large.

The effectiveness of the technique is shown in the experimental results of fig. 6 . For a collimated wave normally incident at $G_{1}$ (fig. 5), the zero (fig. 6a) and corrected first order (fig. 6b) of $\mathrm{G}_{2}$ as viewed by a shearing interferometer are shown. The correction is quite complete.

The author wishes to thank Professors E.N. Leith and J. Upatnieks for helpful discussions during the course of this work. The generous support of the National Science Foundation (Grant Nos. GK-31636 and GK-42361) is greatly appreciated.

\section{References}

[1] E.N. Leith, R. Alferness and S. Case, Opt. Commun. 11 (1974) 204.

[2] E.N. Leith, A. Kozma, J. Upatnieks, J. Marks and N. Massey, Appl. Opt. 5 (1966) 1303.

[3] E.N. Leith and B.J. Chang, Appl. Opt. 12 (1973) 1957. 